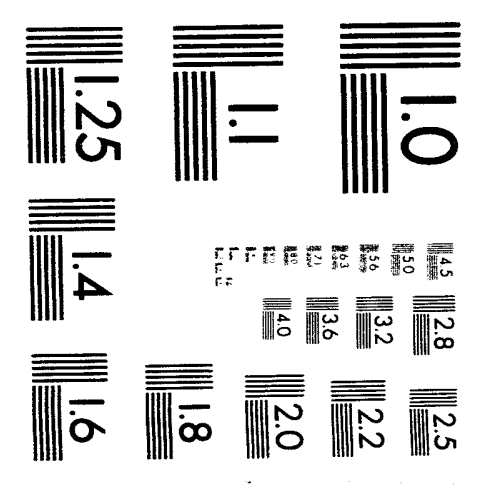



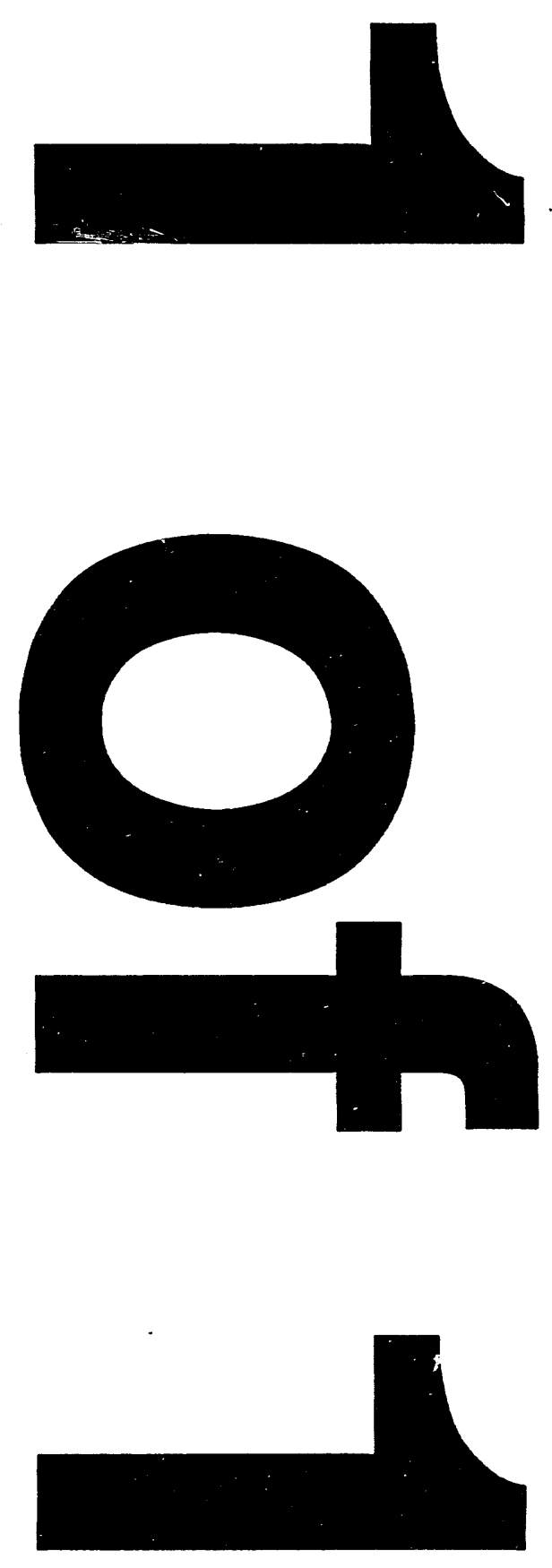


\title{
USE OF OPEN SYSTEMS FOR CONTROL, ANALYSIS, AND DATA ACQUISITON OF THE DIII-D TOKAMAK
}

\author{
by \\ P.A. HENLINE
}

This is a preprint of a paper to be presented at the 15th IEEE Symposium on Fusion Engineering, October 11-15, 1993, Cape Cod, Massachusetts, and to be printed in the Proceedings.

Work supported by U.S. Department of Energy

Contract DE-AC03-89ER51114

GENERAL ATOMICS PROJECT 3466 OCTOBER 1993

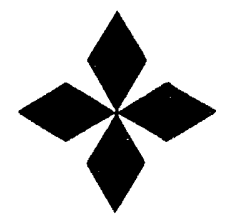

\section{GENERAL}




\title{
USE OF OPEN SYSTEMS FOR CONTROL, ANALYSIS, AND DATA ACQUISITION OF THE DIII-D TOKAMAK
}

\author{
P. A. Henline \\ General Atomics \\ P.O. Box 85608, San Diego, California 92186-9784
}

\section{ABSTRACT}

For the past several years, it has been evident that the very old MODCOMP 16-bit computers being used at DIII-D for control and data acquisition were no longer adequate to perform the services needed. In early 1992, the computer systems group began to look seriously into alternate systems to replace these aged MODCOMP systems. The decision was made to investigate 'OPEN' system computers and also to maintain the compatibility with our large usage of CAMAC equipment as the real-time hardware interface. Information about the needs for real-time capabilities and 'OPEN' systems ability to meet these needs will be discussed. The needs include hardware requirements, operating system software which has known response rates, interconnectability and access of data from other workstations and computers. Some of the parameters and pitfalls of open systems will be discussed as well as the advantages of OPEN systems for use in a real-time environment. Our success at arriving at an OPEN systems solution will be examined.

\section{INTRODUCTION}

This paper will briefly describe the need for new computers at DIII-D, the requirements which were absolutely paramount for a new system, the definition and hoped for gains of moving to an 'OPEN' system, and some of the pitfalls of 'OPEN' systems and some successes of 'OPEN' systems.

One new system is being integrated now in the General Atomics (GA) Fusion Group. It is a MODCOMP model 9087R-64 processor. This is a Motorola 88100 processor chip (to be upgraded to the 88110 ) running MODCOMP's REAL/IX real-time UNIX operating system.

\section{DIII-D BACKGROUND}

In 1975 , plans were made to purchase computers which would support the real-time control and analysis needs for the DIII tokamak. The system selected was a 16-bit minicomputer, manufactured by Modular Computer Corporation, using proprietary hardware and software. Several MODCOMP systems were purchased, put into use, and upgraded during the late 70 s and early 80 s. These MODCOMP systems are used for tokamak control, four MODCOMPs for neutral beam control/data acquisition, and three MODCOMPs for general purpose data acquisition and archiving. In the mid-80s, microVAX and VAX systems were added to control small diagnostics. The VAXes communicate with each other via ethernet, and a Network Systems Hyperchannel is used to connect the MODCOMPs and VAXes. Fig. 1 is a block diagram of the VAX/MODCOMP configuration.

By the late 1980s the electronics industry had changed incredibly, with low price memory and hardware and a whole new paradigm of computing. One of the major changes came in the availability of higher speed CAMAC modules, with high memory capacity. Initial data sizes for DIII-D in February 1989 were a few hundred kilobytes per shot, and shot sizes are now over 80 Mbyte per shot. The MODCOMPs have been outgrown during this period. The old MODCOMPs also can not easily communicate with other computers, access large amounts of memory, are not compatible with many of the new faster pieces of hardware, and have slow bus and CPU speeds.

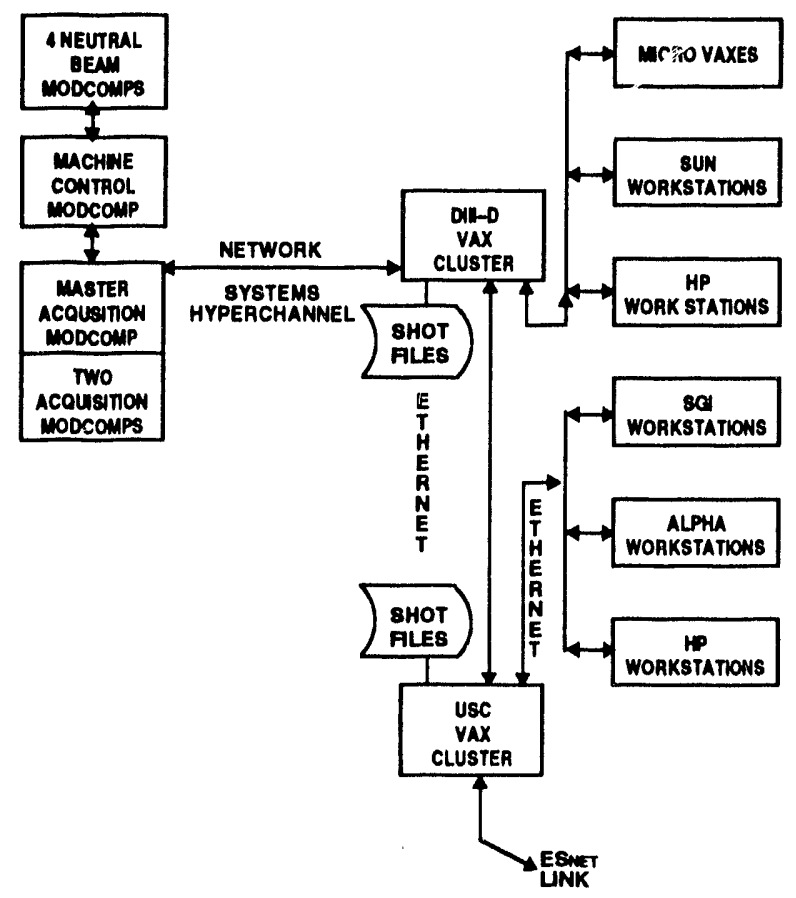

Fig. 1. Back diagram of current VAX/MODCOMP configuration.

Manuscript received October 12, 1993. This is a report of work sponsored by the U.S. Department of Energy under Contract No. DE-AC03-89ER51114.

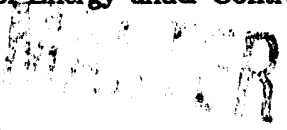




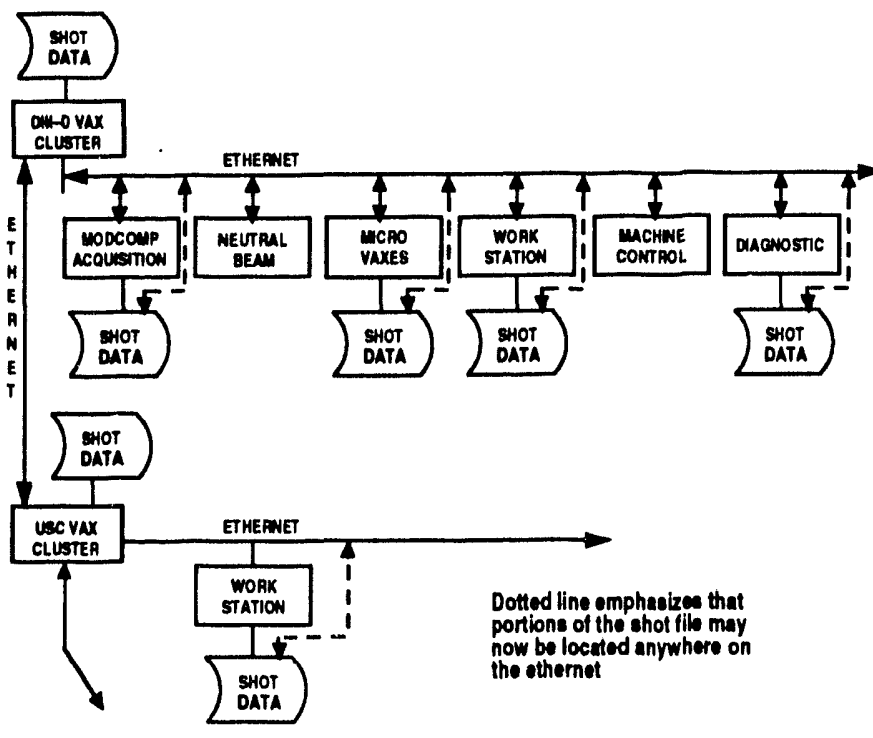

Fig. 2. Block diagram of OPEN systems configuration.

\section{DESIRED CHARACTERISTICS OF A NEW COMPUTER SYSTEM}

There were four major concerns to be met by new computer platforms. The first was that the existing CAMAC equipment must be interfaced to the new system. The DIII-D program has a large investment in CAMAC equipment and a large amount of data is quite adequately collected by this hardware. The second requirement was that the new system must have real-time capabilities to do what the existing systems are doing and more. The third requirement was that the new system should be 'OPEN.' A fourth need was defined as the ability to communicate over a network to existing and future platforms. Fig. 2 is a block diagram which shows the ideal new configuration.

For real-time capabilities, the major hardware need was to be able to interface the CPU to up to ten external interrupts, via some bus which had a reasonable $\mathrm{I} / \mathrm{O}$ rate and was interfaced to real-time hardware modules (such as the VME bus). The software real-time requirements included 128 priority levels for real-time, a fully preemptive kernel, and the ability to load and lock a process into memory. A commitment to POSIX compliance, especially to 1003.4 (the real-time interface), was considered necessary. The commitment was important since POSIX is still in draft form for real-time.

\section{REQUIREMENT THAT THE SYSTEM BE 'OPEN'}

The principal definition of 'OPEN' is that standards be published and followed, for both hardware and software, thus allowing any vendor or person to develop and attach their hardware and run their codes and programs on any system which adheres to the 'OPEN' standards.
The requirement that the system be open came from the need to operate our systems over a relatively long period of time, and the need for the system to grow over that period. The DIII-D tokamak is expected to be in operation for the next six to eight years or longer. Thus open systems must adhere to some industry wide standards to maintain this longevity. Open systems implies that both new hardware and new software can be easily interfaced to existing systems. It also implies that existing software can easily be moved to another open hardware platform and will run. By using open systems, the development and maintenance costs of software can be minimized, and in a competitive marketplace, hardware costs will be reduced, as will the dependence on any one vendor. The control, data collection, and archiving processes for DIII-D now reside in multiple computers, and there is a growing need to use distributed systems to communicate with each other, and to cooperate with each other. National and international collaborations, with diagnostic experiments which are developed remotely, are very common now. The computer systems for these diagnostics need to be integrated quickly and reasonably into the overall scheme. The new open systems should be designed to work in this arena.

\section{ADDITIONAL NEEDS WHICH MAY OR MAY NOT BE CONSIDERED PART OF AN OPEN SYSTEM}

In addition to the above requirements, it was felt that the following items should be part of an 'OPEN' system for DIII- $D$. These include the following:

- FDDI capability

- Open I/O bus, such as SCSI

- Demonstrated hardware upgrade path

- A standard UNIX operating system

- GOSIP compliant

- MOTIF, TCP/IP, NFS

- ANSI C compiler

- FORTRAN and $\mathrm{C}$ with real-time extensions

- X Windows development tools

- Code management system

\section{THE SEARCH FOR THE OPEN SYSTEM}

Another factor influenced the selection of hardware and software. Two basic hardware options exist, a robust CPU with its own development and operating system, or a scaled-down platform which perhaps resides in a real-time crate and runs a scaled down (or down loaded) operating system (such as VX Works). We chose the first type of system because we felt that it was more robust, easier to use for development, had more expandability/capabilities, and ultimately could be used more diversely.

With this basic specification, vendors were contacted for information and later for bids. 


\section{OPEN SYSTEMS AND REAL-TIME NEEDS}

The real-time features needed at DIII-D are primarily a preemptive system and a system which handles external hardware interrupts. An effective set of priority levels also is necessary.

The neutral beam systems run a large number of interrelated tasks. These tasks are started up by a dispatcher which must be able to rely on the tasks being run as needed. Task startup must take place within a known amount of time after the request, thus the system must respond within known time limits.

Once tasks are started up, they must run in a specific sequence, with the most important task getting the priority and system resources which are needed.

System code to handle external interrupts is also mandatory. The neutral beam systems have an abort button. Responding to this button and shutting down hardware is the most important task of the CPU when the button is pressed. External interrupts must receive the highest priority and they must be able to execute code which will stop processes in progress very rapidly.

The system which controls the operation of the tokamak also has important real-time needs. This includes scanning software which must run every second and examine and alarm on 1500 points. Prioritization and preemption are needed for these functions. Also on the control system, there are several external interrupts which are connected to operator buttons which must be acted on quickly. The CAMAC hardware must be initialized quickly after the start button is pressed. And, the operations in progress must be stopped immediately when the crash button is pressed. Certain tasks on the control system are set up to take data at predefined times, and the data must be taken at these times so that the required time history is obtained.

The acquisition system must deal with the CAMAC preshot initialization immediately and do other hardware initialization in the short $10 \mathrm{sec}$ lockout interval before the shot actually fires. Many different codes run during the CAMAC acquisition phase, and these must have priorities suitable to the needs.

There is a broad range of these needed capabilities available under existing open systems. Some systems are absulutely not preemptive. Others have preemption times from $106 \mu \mathrm{sec}$ to $10 \mathrm{msec}$. Most systems have a priority scheme. However, in some cases this is very limited and the user/real-time job has only a few levels to work with, and the rest are reserved for system purposes. Systems with 32 to 64 levels for real-time usage are probably adequate. Hardware for external interrupts seems to be available in some form for most of the buses on open hardware. However, without adequate priority levels and without kernel preemption, handling of external interrupts will not be adequate.
Another area of need for the new DIII-D systems is to communicate with all other systems and share information about the shot setup and progress of the shot. All UNIX systems have TCP/IP which is sufficient, and a third party version of TCP/IP is run on the VAX systems. Communications will be possible, but the response and network delays will have to be considered as the code is developed. Also, data can be shared using network file system (NFS) to make files available to multiple machines. This has proven to be slow so will not be feasible where response is critical.

It was felt that a common set of libraries should be available for codes to run on the real-time systems (especially any type of user computation code), especially graphics and mathematics libraries. These codes would then be able to run on any of the UNIX systems in Fusion since they do not rely on real-time specific features.

\section{'OPEN' AND UNIX}

UNIX has been around for many years since its development. Unfortunately, there have been several major divergences of the operating system which result in significant differences to the user. Individual hardware vendors have had to chose whether to rely on the System V, Berkeley, or MACH versions of the kernel. And, each vendor has added its own individuality to its operating system. Thus, there is currently no single UNIX operating system. Several vendors have formed a group called Common Open Software Environment (COSE) to unify the various versions of UNIX.

There are several evolving versions of UNIX which have major differences between them. SUN is moving from the SUN OS UNIX operating system to their Solaris UNIX operating system. These are quite different from each other. Digital Equipment is phasing out ULTRIX and phasing in OSF $/ 1$. OSF $/ 1$ is based on the standards of the Open System Foundation (OSF) and is a very good implementation of UNIX. Since ULTRIX was developed very early in Digital's efforts to have a UNIX operating system, it has many of its own features and is quite different from OSF/1.

There are many UNIX versions - SUN OS, Solaris, ULTRIX, OSF/1, AIX, HPUX, REAL/IX, and each has some distinct properties to it. The overriding reason for dealing with UNIX is that the differences between these versions of UNIX is smaller than the difference between major operating systems such as UNIX and VAX VMS or UNIX and IBM MVT. And, there is enough similarity that even the casual user can move from UNIX system to UNIX system and work in the OS environment. In many cases, codes, once ported to any UNIX system, are more easily ported to another UNIX system.

\section{'OPEN' AND UNIX AND REAL-TIME}

Since each vendor has made enhancements to its own operating system to achieve real-time functionality, 
these features will be different from vendor to vendor. When POSIX 1003.4 becomes finalized (probably within the next two to four years) each vendor will be able to standardize to the same template.

In order to generate code which is most easily portable, these differences must be recognized and code must be written which separates and encapsulates the nonstandard calls and features. By isolating this code, it can more easily be converted to run on some other realtime platform.

MODCOMP has its own set of real-time routines which are used as part of the UNIX operating system. The MODCOMP UNIX system is a complete kernel rewrite of the basic UNIX operating system.

Unique routines and facilities exist in the following areas [2]:

Subprocess Subsystem

- fully-preemptive kernel with synchronization mechanisms

- fixed-priority based process scheduler for critical real-time processes

- real-time timer mechanisms

- enhanced memory management facilities

Interprocess Communications

- robust common event notification mechanism

- fast binary semaphore mechanism

File Subsystem

- alternative file system architecture that supports preallocation of space and contiguous files

- ability to bypass the buffer cache for file $\mathrm{I} / \mathrm{O}$

- synchronous updates of physical storage devices

- larger logical block sizes

- improved locality of reference

I/O Subsystem

- support for emulating asynchronous $1 / O$ operations

- disk I/O queuing prioritized according to process priority

- direct I/O between a user-level program and a device

- connected interrupts for notifying a user-level process of a hardware interrupt

A number of these features have been used implicitly and others are called explicitly. For instance, the routine "nanosleep" has been used in the CAMAC handling routines. And calls which make a process a real-time process and set priority levels have been used.

\section{OUR SUCCESS AT 'OPEN'}

Totally portable code cannot be developed for the UNIX operating system (or other operating systems which run on multiple vendor platforms) at this time. The additional needs of code to perform real-time tasks further limits generality. Good coding practices and the choice of general purpose routines over machine specific routines will result in the most portable code. This includes use of standard $\mathrm{C}$ and/or FORTRAN constructs. Use of any machine assembly code should be avoided if possible.

The code being developed for the new systems at GA is following these guidelines. Most of the code compiles on a standard $\mathbf{C}$ compiler (including on IBM PCs where much of the early development was done). The personnel who are working with the MODCOMP system have developed into UNIX system experts. This expertise carries over to UNIX on all of the various hardware platforms at GA since there are major similarities.

Our success at OPEN on the real-time systems is partially tied to our success with UNIX on the various UNIX platforms already in use in the Fusion Division at GA. The major overall concern is to develop code which is platform independent as much as possible. A particular concern is graphics libraries and mathematics libraries. Several graphics libraries (at least four are in use on different platforms) and one major mathematics library is in use. Site licenses or quantity discount prices must be arranged if this software is to be available on every UNIX computer and workstation. Additionally, this software should also be available on the VAX platform. And, the same software should be used on the real-time platforms.

If new hardware platforms from other vendors are purchased for later systems, the major portion of the software will be portable. Probably $20 \%$ to $30 \%$ will need specific effort to convert.

Thus it is felt that we have achieved as much of the portability and openness as is possible at the current time.

This move to a more open real-time operating system should make it easier to develop code in the future for similar systems. Costs of hardware should be less expensive than with proprietary systems. Future new systems and maintenance of existing systems should be reduced, but many new capabilities will be available now and in the future as CPUs become more powerful and less costly.

\section{ACKNOWLEDGEMENT}

I would like to thank the personnel who helped develop specifications and analyze bids and equipment for the open systems platform - John Cummings, Bill McHarg, Paul Thurgood, and Jim Vanderlaan.

\section{REFERENCES}

[1] P.A. Henline, private communication, July 17, 1992.

[2] "Concepts and Characteristics, REAL/IX Operating System, Open Architecture Systems." Ft. Lauderdale, FL: Modular Computer Systems, Inc., 1991. 

